\title{
A Reverse-Engineering Approach for the Management of Product Geometrical Variations during Assembly
}

\author{
Jean-Loup Gregorio ${ }^{1,2}$, Claire Lartigue ${ }^{1}$, François Thiebaut ${ }^{1}$, Hugo Falgarone ${ }^{2}$ \\ ${ }^{1}$ LURPA, ENS Cachan, Univ. Paris Sud, Université Paris Saclay, Cachan 94235, France \\ ${ }^{2}$ Airbus Group Innovations, Suresnes 92152, France
}

\begin{abstract}
One of the major challenges for the aerospace industry is to manage a range of complex products during their manufacturing and assembly process. Digital Mock-Ups (DMU) are nowadays extensively used as supports of information during the aforementioned phases. In this paper we introduce the concept of updated DMU which actually reflects the product being assembled. For this purpose, the nominal product geometry needs to be updated in order to reflect the as-built geometry of the product's components at a given step of its assembly. The geometry of the next components to be assembled, called interface components, are consequently updated in order to adapt to geometrical variations, while the rest of the DMU stays in its original as-designed configuration. A comprehensive method to update the DMU from its as-designed configuration to reflect the actual configuration of the product being assembled is thus proposed. To this end, a framework inspired from the ReverseEngineering field is developed, then assessed thanks to a simple case study.
\end{abstract}

Keywords: Reverse-Engineering; Product manufacturing; As-built model; Digital Mock-Up. 


\section{Introduction}

In order to speed-up the manufacturing and assembly processes, physical mock-ups of aeronautical products have been gradually replaced with their digital counterparts, leading to the concept of product virtual representation also known as Digital Mock-Up (DMU). The DMU typically consists in a detailed 3D representation of the product's geometry but also contains information related to manufacturing and assembly processes such as tolerances, machining programs, assembly planning, inspection procedures, and so on. During manufacturing and assembly phases, the DMU stands for the reference geometry of the product. More particularly, the geometry of the elements constituting the DMU is described in the product as-designed configuration, which means the components are without geometrical deviations and in their theoretical position.

Tolerancing methods aim to confine product geometrical variations within an admissible interval so that it satisfies the functional requirements expressed in the DMU. Geometrical constraints are thus established between elements to be manufactured or assembled, and specified elements of the initial product. Tolerancing methods are often coupled with Computer Aided Tolerancing Tools [1] relying on the DMU, enabling worst case and/or statistical tolerancing of rigid assemblies. Improvements have been made all along the years in order to provide more realistic tools for aeronautical assemblies [2], which are considered flexible due to large size and low thickness components.

Despite the use of tolerancing methods and tools, the management of product geometrical variations does not allow an accurate prediction of the entire geometry of the actual product at each step of the assembly process. Aeronautical products are typically composed of thousands of parts, often made of composite materials and presenting a high length-to-thickness ratio, which makes geometrical variation estimation very tedious. As a result, unpredicted discrepancies inevitably appear between the actual product and its reference digital model [3][4]. Consequently, features of already assembled components do not constitute a valid basis to continue the assembly process.

In order to avoid cost-ineffective adjustment operations or the generalized use of jigs, some authors propose to use techniques derived from Reverse-Engineering in order to adapt to discrepancies by manufacturing custom-made components [3][4]. The geometry of specific interface components is customized so that they perfectly fit within the available space. More particularly, geometrical data are captured in the shop floor assembly station, in order to adjust the interface component CAD model. Interface components are afterwards manufactured then assembled.

In this paper, we introduce the concept of hybrid representation of the updated DMU in order to reflect the actual geometry of a product during its assembly. The updated DMU is composed of as-built components, describing the actual configuration of the product being assembled, interface components and as-designed components. The use of such a DMU allows a more comprehensive management of the 
product's geometrical variations by establishing bi-directional relations between the physical product and its virtual model, often referred to as digital twin. In a broader scope, some authors show that the establishment of such relations between physical parts and their virtual models would enable a more efficient execution of the product activities all along its lifecycle [5].

Obtaining such an updated DMU, reflecting the actual geometry of the product including its geometrical deviations, is not straightforward and serious difficulties must be overcome. Within the context of Reverse Engineering, only a few papers aim at reconstructing models of existing products in order to underline geometric changes with a preexisting DMU. In most cases, the information - geometrical or linked to the product use - extracted from an eventual preexisting DMU only serves as an initial estimate of the product to be reconstructed [6]. In [7, 8] authors decompose the initial CAD model of the product into a set of tessellated components that can be easily matched to measured data. These components are first recognized in the CAD model, along with their properties and global relations in order to facilitate the matching. Once the matching between tessellated components and measured data is carried out, a registration algorithm is used in order to calculate the as-built pose of the CAD model components, controlling the compliance of the product with respect to corresponding dimensional tolerances. Other authors propose to use CAD templates of mechanical parts $[9,10]$ containing a set of design features which parameters can be modified thanks to data extracted from the real world (mainly 3D measurements). Such a template-based reverse engineering method consists in exploiting a parametric description of the object in order to retrieve a meaningful digital representation. An extension of the previous method to mechanical products is proposed in [11]. The main advantage of this approach is that it eventually results in a fully editable CAD model which is consistent with the original design intent which mostly makes it suitable for redesign purposes only.

This paper gives a detailed description of our proposition of an updated DMU based on a hybrid representation. Contrary to the as-designed model, the geometry of the updated product reflects product geometrical deviations. Based on this information, the geometry of the components to be assembled is also updated in order to impact the manufacturing and assembly activities based on the DMU. An illustration of our Reverse-Engineering framework for updating the as-designed components to as-built and interface components using data acquired from optical measurements and the available engineering knowledge is also presented.

\section{From the as-designed DMU to the updated DMU}

Our concept of an updated DMU based on a hybrid representation, is illustrated through a simple case study based on a product consisting of four assembled parts: a flanged box (Meccano ${ }^{\circledR}$ part $\left.n^{\circ} 236\right)$, two triunions (Meccano ${ }^{\circledR}$ part $\left.n^{\circ} 126\right)$ and a perforated strip (Meccano ${ }^{\circledR}$ part $\left.n^{\circ} 4\right)$. The as-designed DMU (Fig. 1) is constructed 
using the CAD software CATIA $®$ V5 and the part's engineering drawings. As it is often the case, and for a better understanding, fixturing parts are not represented in the DMU.

Global references are defined as a set of three planes, named A, B and C, representing respectively the primary, secondary and tertiary references. Geometrical requirements are defined as a localization tolerance that aims to position a set of three holes regarding to the global references (Fig. 1). Here tolerancing aims to confine the position of each hole axis into the cylindrical region specified by the tolerance value.

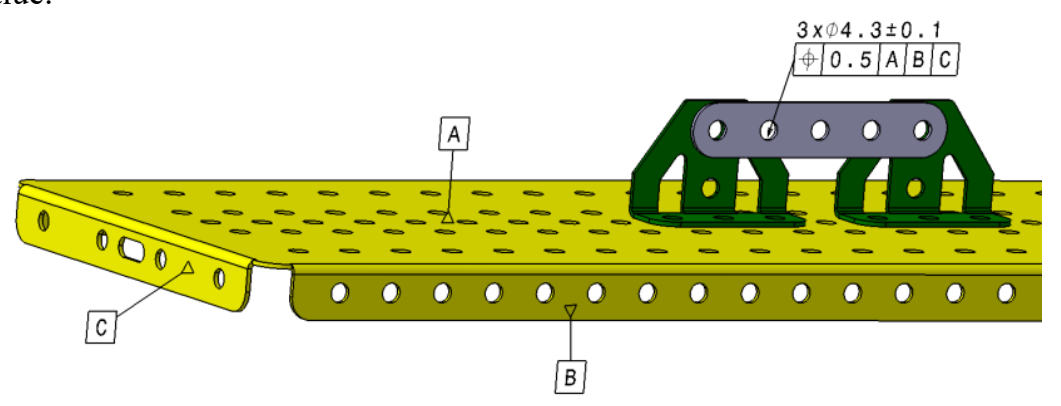

Fig. 1. Partial representation of our case study functional requirements

In the proposed scenario, the first three parts, i.e. the yellow box and the two green triunions are manufactured then assembled (Fig. 2.b.). The geometry of the actual product is acquired, and thus updated in the DMU thanks to our method in order to reflect the as-built configuration of the already assembled components. Based on the as-built geometry, the geometry of the last component is updated so that, once assembled, the functional requirements of the final product are met. More particularly, the position of the pattern of holes is updated so that the position of the specified hole axes in the desired tolerance interval.

With the classical manufacturing approach in the aerospace industry [1, 2, 3, 4], a specific tolerance is allocated to each functional surface or local reference of the product. In the best case, a statistical or worst case calculation is performed in order to find a tradeoff between the overall production costs induced by these tolerances and the assurance that functional requirements will eventually be met. Very often, these tolerances cannot be directly assessed and the as-designed DMU serves as reference model to transpose geometric constraints between elements to be manufactured and global references into much simpler constraints where only local references are involved. For example, the first fixturing hole axis is assumed to be in the same position than in the as-designed DMU, and the second one is drilled so that the final part remains parallel to reference A. As discrepancies tend to appear between the actual product and the as-designed DMU, this scenario often leads to several mandatory additional adjustment steps in order to guarantee that the final assembled product eventually meets the specified functional requirements (Fig. 1). 
The situation of the local references, here the fixturing hole axes (L1 and L2) and the contact plane with the triunions (L3), relatively to the global references, and how it impacts the product's functional requirements is the key concern of our approach. Each local reference is expressed in the frame defined by the global references together with the product's functional requirements. In the as-designed DMU (Fig. 2.a.), the local references (L1, L2 and L3) are theoretically positioned regarding to global references. This configuration does not account for the actual geometry of the product.

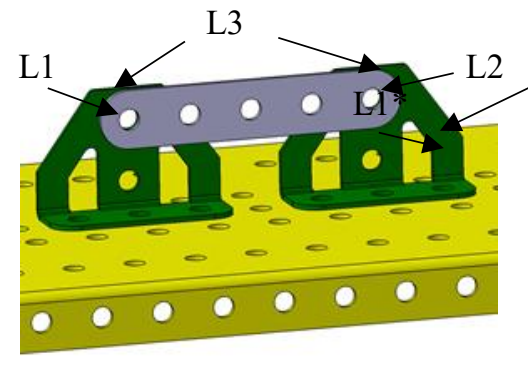

(a) As-designed DMU

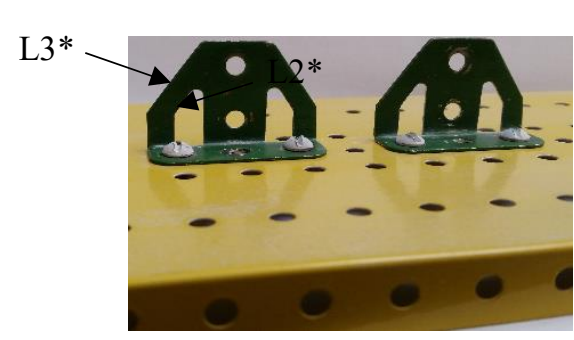

(b) Built product

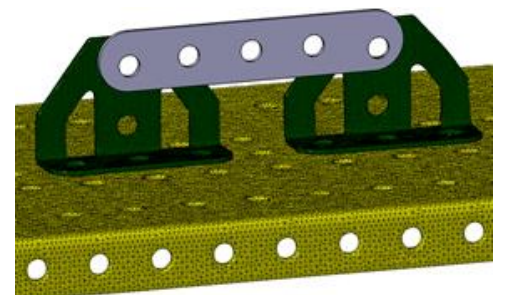

(c) Updated DMU

Fig. 2. The product as-designed model (a) is updated to reflect the built components at a given assembly step (b), following manufacturing and assembling operations are also impacted (c)

We use a Reverse-Engineering based method to update the product's already assembled components so that they reflect the actual geometrical configuration of the product at a given step of the assembly process (Fig. 2.b.). The updated as-built components integrate the geometrical variations between the as-designed model and the actual product. As a result, the situation of the local references in the frame defined by global references is known by the user.

The newly defined situation of the local references $(\mathrm{L} 1 *, \mathrm{~L} 2 *$ and $\mathrm{L} 3 *)$ is used to adapt the geometry of the next component to be assembled, called the interface component, so that the respect of the product's functional requirements is ensured (Fig. 2.c.). Therefore, following manufacturing and assembly operations, which are based on the interface component's geometry, are impacted. 
In the case study, the DMU only contains as-built components and interface components. Nevertheless, we could imagine a scenario where some additional components would be assembled to the interface part. In this scenario, the previously specified hole axes would serve as local references in order to continue the assembly process. As the geometry of the interface part has been updated in order to meet the product specified functional requirements, these local references constitute a valid basis to continue the assembly. Therefore the to-be-assembled component geometry would not require to be updated and the assembly process could be carried on accordingly to the information initially contained in the as-designed DMU. As a result, the updated DMU would contain as-build components, interface components and as-designed components.

In the general concept of updated DMU based on a hybrid representation, asbuilt components coexist with interface components and as-designed components. As-designed components are components which are already present in the initial asdesigned DMU and which have not been assembled yet. As-built components correspond to components that have been manufactured and whose geometry has been acquired and updated in the DMU in order to integrate their geometrical deviations. Interface components correspond to components that have not already been manufactured and assembled and whose design has been updated in order to be assembled to the as-built components so that the product's functional requirements are met.

In the next section an overview of our Reverse-Engineering method is presented. Our method allows the updating of the product's as-designed configuration to as asbuilt configuration as presented previously (Fig. 2.b.). The different steps of our method are individually assessed.

\section{Illustration of the proposed approach}

The proposed method aims to update components of an initial as-designed DMU to the as-built configuration which reflect the actual state of a given product.

The first three components are assembled and digitized using a laser plane sensor (Kreon Zephir K2 25) mounted on a CMM. The different steps of our reverse-engineering framework (Fig. 3) are implemented in Python and Matlab ${ }^{\circledR}$ and run on a laptop with a $2.3 \mathrm{GHz}$ CPU and $8 \mathrm{~GB}$ of RAM. The as-built DMU is reimported in CATIA ${ }^{\circledR}$ V5.

Data acquired from optical measurements are used as a representation of the real product. These data need to be processed, relying on the as-designed geometry and user's knowledge about the product, in order to retrieve the product's components in the as-built configuration. Our method consists in five steps: data preprocessing, global registration, segmentation, local registration and 3D modelling. 


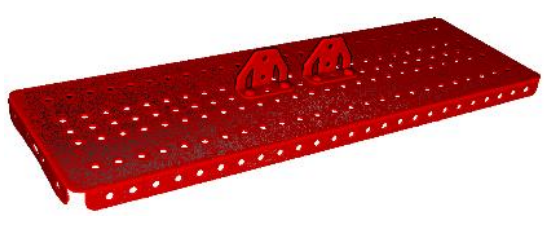

(a) Data preprocessing

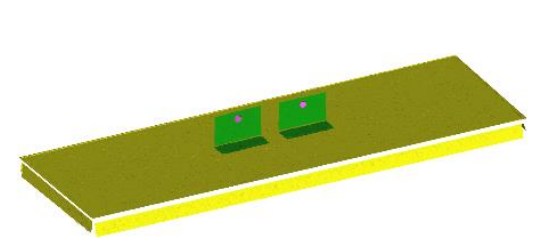

(c) Segmentation

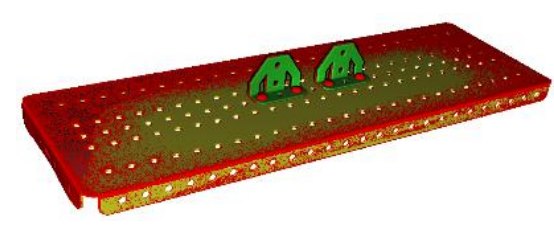

(b) Global registration

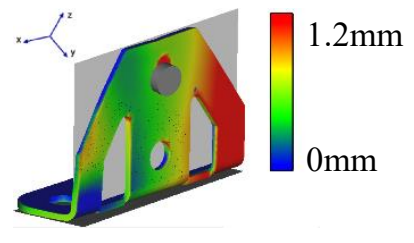

(d) Local registration
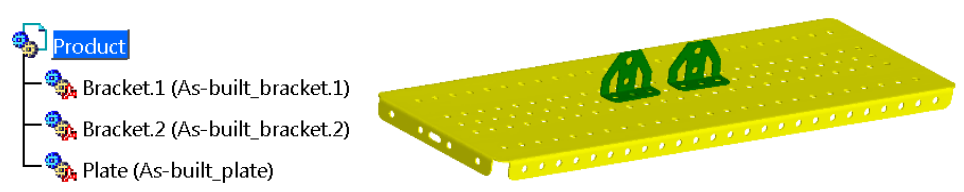

(e) 3D modelling

Fig. 3. Illustration of the Reverse-Engineering method on a simple assembly

The component surface geometry is acquired using an optical measuring system which delivers a numerical representation of the geometry as a point cloud. The obtained raw point cloud, generally dense, non-homogeneous and highly noisy, is not readily usable for our application, and needs to undergo a data preprocessing step (Fig. 3.a.).

During the second step (Fig. 3.b.), the point cloud is registered to the as-designed geometry. In order to establish a meaningful correspondence between the data and the model, engineering knowledge about the product's functional requirements is used. In our case, we use the knowledge about the reference surfaces of our model, i.e. the global references, in order to register the point cloud to our reference frame so that geometrical deviations, extracted during further steps, are also expressed in this reference frame.

During the segmentation step (Fig. 3.c.), the actual situation of the product's components and elements of interest, i.e. local references, is identified from the point cloud. The first goal specifically aims to divide the point cloud into a set of features representing the assembly components.

Geometrical deviations between the segmented elements and their as-designed equivalents are then characterized, and a step of local registration is next performed 
in order to integrate these deviations into the initial model (Fig. 3.d.). The position, the orientation and also the form of the components are adjusted in order to minimize an error metric between the geometry and the measured point cloud.

Finally, the resulting geometry is exported into a commercial CAD software. The global model consistency is checked and adjustments are made in order to guarantee that no gaps or intersections are found in the final as-built geometry (Fig. 3.e.).

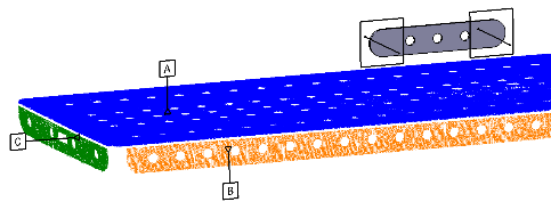

(a) Part before drilling

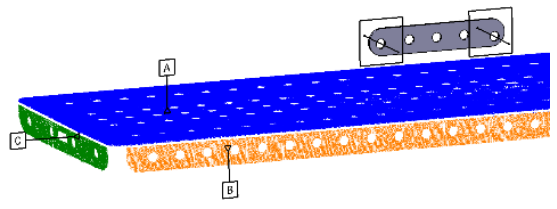

(b) Part after drilling

Fig. 4. Adapting the drilling operation in order to meet functional requirements

Once the component's as-built geometry has been reconstructed, the situation of the local references regarding to the global references can be deduced in order to impact further manufacturing and assembly operations. In the proposed case study, the drilling operation to manufacture the fixturing holes is here impacted (Fig. 4). Indeed, the design of the interface component has been updated so that functional requirements of the assembly are met without the need of adjustment operations. The component is positioned in the global reference frame and features corresponding to local references, here the two fixation hole axes, are updated thanks to the information extracted from the as-built geometry.

\section{Results and discussion}

In this paper, we proposed to use a hybrid representation of an updated DMU in order to provide a better management of geometrical variations during assembly. The originality of our approach is that the updated DMU not only reflects the actual geometry of the product, including its geometrical deviations, but also serves to adapt manufacturing and assembly operations so that the final product's functional requirements are met without the need of cost-ineffective adjustment operations.

The Reverse-Engineering method presented in this paper provides theoretical tools necessary to update a given DMU from its as-designed configuration to its hybrid updated configuration. The obtained Digital Mock-up reflects on the one hand the as-built geometry of the product's already assembled components, including geometrical deviations, and on the other hand the geometry of the components to be manufactured and assembled, which is updated in order to adapt to existing geometrical deviations. 
However, interfacing as-built components with as-designed components is not straightforward and raises some interesting questions. Interfaces between as-built components and as-designed components involve on the one hand surfaces with geometrical deviations and on the other hand surfaces without geometrical deviations. In this case, deducing the assembly behavior resulting from the contact of these surfaces does not admit a simple solution and would strongly depend on the underlying assembly model.

A solution for a simple test case is proposed in this paper but both theoretical and practical developments will be needed in order to adapt it for industrial aeronautical products.

Based on the results already presented in this paper a roadmap for future developments is established. Future work will primary focus on a better modeling of the product's geometrical behavior and a better interface modeling between as-built and as-designed components. To this end, integrating graph based and skeleton based approaches to our Reverse-Engineering method seems promising.

\section{Acknowledgments}

The research work reported here has been carried out in partnership between Airbus Group Innovation and the LURPA - ENS Paris-Saclay.

\section{References}

[1] Marguet, B., Chevassus, N., Falgarone, H., \& Bourdet, P. Geometrical behavior laws for computer aided tolerancing: Anatole a tool for structural assembly tolerance analysis. In Eighth International Seminar on Computer-Aided Tolerancing, Charlotte, NC, pp. 301-310, (2003).

[2] Falgarone, H., Thiébaut, F., Coloos, J., \& Mathieu, L. Variation simulation during assembly of non-rigid components. Realistic assembly simulation with ANATOLEFLEX software. Procedia CIRP, 43, 202-207. (2016).

[3] Fu, P. Reverse Engineering in the Aerospace Industry. In Reverse Engineering, pp. 157-175. Springer, London (2008).

[4] Gómez, A., Olmos, V., Racero, J., Ríos, J., Arista, R., \& Mas, F. Development based on reverse engineering to manufacture aircraft custom-made parts. International Journal of Mechatronics and Manufacturing Systems, 10(1), 40-58 (2017).

[5] Schleich, B., Anwer, N., Mathieu, L., \& Wartzack, S. Shaping the digital twin for design and production engineering. CIRP Annals, 66(1), pp. 141-144, (2017).

[6] Bey, A., Chaine, R., Marc, R., Thibault, G., \& Akkouche, S. Reconstruction of consistent 3D CAD models from point cloud data using a priori CAD models. In ISPRS Workshop on Laser Scanning, (2011)

[7] Bosché, F. Automated recognition of 3D CAD model objects in laser scans and calculation of as-built dimensions for dimensional compliance control in construction. Advanced engineering informatics, 24(1), 107-118 (2010). 
[8] Erdős, G., Nakano, T., \& Váncza, J. Adapting CAD models of complex engineering objects to measured point cloud data. CIRP Annals-Manufacturing Technology, 63(1), pp. 157-160, (2014).

[9] Durupt, A., Remy, S., Ducellier, G., \& Guyot, E. A new reverse engineering process, the combination between the knowledge extraction and the geometrical recognition techniques. In Computers \& Industrial Engineering, 2009. CIE 2009. International Conference on, pp. 1367-1372, IEEE (2009).

[10] Buonamici, F., Carfagni, M., Furferi, R., Governi, L., Lapini, A., \& Volpe, Y. Reverse Engineering of Mechanical Parts: a Template-Based Approach. Journal of Computational Design and Engineering, (2017).

[11] Bruneau, M., Durupt, A., Vallet, L., Roucoules, L., \& Pernot, J. P. A three-level signature by graph for Reverse Engineering of mechanical assemblies. In Tools and Methods for Competitive Engineering (Aix-en-Provence: 16: 2016), pp. 669681 (2016). 